\title{
PRÁCTICAS DE RECURSOS HUMANOS Y SU IMPACTO EN LOS RESULTADOS DE INNOVACIÓN: UNA REVISIÓN TEÓRICA
}

Human Resources Practices And Their Impact on Innovation Performance: A Theoretical Review

\section{Naiara Escribá Carda Francisco Balbastre Benavent Teresa Canet Giner}

Resumen: El presente artículo tiene como objetivo la realización de una revisión teórica con la finalidad de identificar las prácticas de Recursos Humanos más analizadas respecto a los resultados de innovación y, como resultado, poner de manifiesto otras variables no consideradas hasta la fecha pero que también tengan incidencia en dicha relación. Así pues, autonomía, participación, formación, sistemas de recompensas, trabajo en equipo, reclutamiento y selección, además del desarrollo de carrera profesional, afectarían positivamente los resultados de la innovación a través de la creatividad y la gestión del conocimiento. La estructura y cultura organizativa jugarían un papel moderador en esta relación. Por lo que la contribución básica reside en el planteamiento de un modelo teórico integrador de las variables que relacionan las prácticas de recursos humanos y los resultados de innovación así como de aquellas otras que resultan mediadoras y moderadoras entre ellas.

Palabras claves: Prácticas de recursos humanos, resultados de innovación, variables mediadoras y moderadoras. 
Abstract: The paper presented here aims at developing a theoretical review in order to identify those Human Resources Practices linked to innovation performance that have been most analysed by the specialised literature and, as a result, reveal other different variables affecting this relationship that have been overlooked to date. The study reveals that autonomy, participation, training, compensation, teamwork, recruitment and selection, as well as career development, have a positive impact on innovation performance mediated by creativity and knowledge management. Also, organisational structure and culture play a moderating role in this relationship. Thus, the main contribution of this paper lies in presenting an integrative theoretical model about those variables relating Human Resources Practices and innovation performance as well as those factors mediating and moderating this relationship.

Keywords: Human Resource Practices, innovation performance, mediators \& moderators variables.

\section{Introducción}

Los recursos humanos son considerados el activo más valioso de las organizaciones. Además, diversos autores afirman que facilitan la obtención de un mejor resultado final para estas (Pfeffer, 1998; Wimbush, 2005). Por otra parte, la innovación, entendida de forma general como un proceso que implica la introducción de un nuevo, o significativamente mejorado, producto (bien o servicio) (OECD-Eurostat, 2005), está configurándose como una de las estrategias empresariales más adoptadas por las organizaciones para hacer frente a los cambios de los entornos competitivos actuales. 
Desde la literatura tradicional de Dirección de los Recursos Humanos se ha puesto especial énfasis en la identificación de Prácticas de Recursos Humanos (PRH en adelante) susceptibles de mejorar los resultados organizativos. Cabe señalar que cuando hablamos de PRH hacemos referencia a la determinación de las necesidades de reclutamiento, selección, formación, sistemas retributivos, evaluación y atención a las relaciones laborales, de salud y seguridad y justicia (De Cieri y Krammar, 2008; Dessler, 2011). Se parte de la premisa de que la adopción de determinadas estrategias requiere de la aplicación de PRH que son diferentes de las que serían necesarias para estrategias distintas o alternativas (Jackson y Schuler, 1995; Schuler y Jackson, 1987a y b; Schuler y Jackson, 1988) lo cual nos lleva a considerar las PRH desde una perspectiva dinámica y contingente. La literatura sobre Recursos Humanos había expresado vagamente su interés en determinar qué prácticas están vinculadas con la estrategia de innovación y cuáles son las que tienen impacto sobre los resultados innovadores, ya que tradicionalmente se ha centrado en analizar el impacto de estas sobre la performance organizativa. Por este motivo, en la última década han ido en aumento los estudios que analizan las PRH que conducen a obtener resultados innovadores (Leede y Kees, 2005; Looise y Van Riemsdijk, 2004).

Hay que tener en cuenta que los modelos de innovación expresan la necesidad de aplicar determinadas PRH (Tang, 1998); el presente artículo tiene como objetivo analizar desde un punto de vista teórico la literatura sobre Recursos Humanos, así como la relativa a la innovación tanto en su vertiente de proceso como de resultado con la finalidad de identificar las PRH más analizadas en relación a los resultados de innovación. Se plantea aquí un modelo teórico, se identifican otras variables que afectan esta relación y, por último, se pasa a plantear futuras líneas de investigación al respecto a esta nueva tendencia de investigación en la literatura de Recursos Humanos. 
Esta revisión se encuentra estructurada de la siguiente manera. La primera sección del trabajo ofrece una fundamentación teórica y empírica obtenida a través de la revisión bibliográfica de los principales enfoques de recursos humanos e innovación desde una perspectiva tradicional para dar paso al análisis de los principales trabajos que a través de una representación más novedosa analizan la relación entre las PRH y la estrategia de innovación y los resultados innovadores. Seguidamente, se analizan otros trabajos en los que se plantea la existencia de otras variables que tienen impacto en la relación estudiada. Por último, se plantea un modelo teórico surgido de la anterior revisión de la literatura, y se plantean las principales limitaciones y futuras líneas de investigación.

\section{Diferentes enfoques de recursos humanos y performance organizativa}

La literatura tradicional sobre Recursos Humanos, como anteriormente se ha apuntado, se ha centrado en analizar qué PRH tienen un mayor impacto sobre la performance organizativa (ver revisiones: Becker y Gerhart, 1996; Gurbuz y Ibrahim, 2011; Huselid, 1995; Vlachos, 2008). A continuación se explican los principales enfoques que adoptan estos estudios para en puntos posteriores presentar la literatura más relevante en cuanto a la relación entre PRH y la estrategia y resultados de innovación, objeto de la presente revisión.

De acuerdo con la categorización de (Jackson, Schuler, y Rivero, 1989), (Brewster, 1995; 1999) y (Delery y Doty, 1996) se pueden identificar cuatro perspectivas básicas en cuanto a la relación entre las PRH y la performance organizativa; estas se refieren a la universal, la contingente, la configurativa y la contextual. A continuación las explicamos brevemente: 
La universalista es considerada la perspectiva más simplista de las cuatro. Se parte de la premisa inicial de que existe una relación lineal entre las variables que puede extenderse a todas las organizaciones (Delery y Doty, 1996).

Por lo tanto, derivado de los numerosos estudios en este ámbito existe una descripción de "best practice" o prácticas superiores que pueden afectar de forma independiente o conjunta a la performance empresarial (Becker y Gerhart, 1996). Algunas de estas prácticas, de acuerdo con Delery y Doty (1996) se refieren a la oportunidad de desarrollo profesional, planes de formación, evaluación del rendimiento, políticas de comunicación, participación en beneficios, seguridad en el trabajo y a la descripción de los puestos de trabajo. Hay que añadir que a esta corriente de definición de prácticas superiores se han unido muchas críticas por considerar que algunas prácticas pueden entrar en conflicto con otras.

El enfoque universalista estudia las prácticas desde un punto de vista exclusivamente aditivo (Becker y Gerhart, 1996; Osterman, 1994; Pfeffer, 1994). En relación con los demás enfoques o perspectivas podemos afirmar que este enfoque se caracteriza por la escasa o deficiente argumentación teórica (Martín-Alcázar, Romero-Fernández, y Sánchez-Gardey, 2004).

En respuesta a las limitaciones de este enfoque inicial aparecieron otras aportaciones que apoyaban la necesidad de considerar la coherencia externa y consistencia interna de las prácticas. En definitiva, la respuesta a este enfoque universalista toma dos direcciones no necesariamente excluyentes entre sí, más bien complementarias: el enfoque contingente y el configurativo.

El enfoque contingente niega la existencia de las best practices y por tanto critica que la aplicación de estas aporte un mayor desempeño bajo cualquier circunstancia (Delery y Doty, 1996). Desde este enfoque se propone un modelo basado en la interactividad, es 
decir, la relación entre las variables no es lineal sino que depende de variables de contingencia para la gestión de los RRHH (Martín-Alcázar, Romero-Fernández, y Sánchez-Gardey, 2005; Venkatraman y Prescott, 1990). Por lo tanto, las prácticas más adecuadas dependen de factores del contexto de la función de recursos, tanto internos como externos a la organización globalmente considerada. Desde este enfoque se desprende la necesidad de que exista una coherencia o congruencia externa entre las PRH y la estrategia corporativa. A modo de ejemplo y siguiendo la teoría contingente de Miles y Snow (1978) el grado de innovación puede considerarse una variable contingente a través de la cual se puede clasificar la posición competitiva de las organizaciones (p. ej. altamente innovadoras: prospectivas, moderadamente innovadoras: analizadoras y raramente innovadoras: defensivas). Dado el ejemplo, se entiende que en función del tipo de variable contingente se clasifica a las empresas en distintos tipos y, por lo tanto, cada tipología se caracterizaría por la aplicación de determinadas PRH.

Desde el enfoque configurativo se pone de manifiesto que para obtener una buena performance existen configuraciones de prácticas que son internamente consistentes, aunque la relación entre las prácticas y los resultados no es lineal (Camelo, Martín, Romero, y Valle, 2004; Delery y Doty, 1996; Martín-Alcázar et al., 2005; Venkatraman y Prescott, 1990) sino que las interdependencias existentes entre las prácticas pueden provocar efectos sinérgicos tanto positivos como negativos, ampliando o reduciendo el efecto combinado de las mismas.

Los trabajos que podríamos encuadrar dentro del enfoque configurativo proponen sistemas de RRHH internamente consistentes, en lugar de prácticas aisladas o independientes. Un problema propio de los trabajos encuadrados en este enfoque configurativo se refiere a que asumen la hipótesis contingente, por lo que más que recomendar un sistema de prácticas 
"universal" presentan diferentes sistemas más o menos apropiados a una estrategia o contexto determinado. A modo de ejemplo, Delery y Doty (1996) establecen dos sistemas de "empleo" basándose en las siete prácticas comentadas anteriormente como "best practices", uno externo orientado al mercado y otro interno. Así, el orientado al mercado se caracteriza por contratar de forma externa, por tener pocos programas de formación, evaluar el rendimiento en función de los resultados, baja comunicación y seguridad en el empleo y las descripciones de los puestos de trabajo son ampliamente de tallas. Por otra parte, el sistema con orientación interna se caracteriza por la existencia de un mercado de trabajo interno, por una amplia socialización y formación de los empleados, los sistemas de evaluación tienen un mayor carácter de desarrollo más que de evaluación de resultados, se tiene una mayor consideración de las propuestas de los empleados, existe una mayor seguridad en el empleo y las descripciones de los puestos suelen ser menos detalladas.

Como ya decíamos, estos dos últimos enfoques no solo no son excluyentes sino que son complementarios. El primero (el contingente) se centra básicamente en los aspectos externos a la función de RRHH y sus efectos sobre la misma, mientras que el segundo (el configurativo) se centra en los aspectos internos y en las interacciones entre las distintas PRH.

Más recientemente se introduce una nueva perspectiva de análisis de la Dirección de RRHH, la contextual, que a diferencia de los otros enfoques en los que el entorno, en el mejor de los casos, se consideraba como una variable de contingencia, se defiende la necesidad de expandir el concepto de dirección estratégica de recursos humanos, para explicar cómo influye en su contexto externo y en el contexto organizativo en el cual las decisiones directivas son tomadas (Brewster, 1999). Las estrategias y PRH se analizan y explican a través de su contribución a los resultados organizativos y por la influencia en otros aspectos de la 
organización y de sus efectos en el contexto (Martín-Alcázar et al., 2005). Así pues, el enfoque contextual propone una explicación que excede el nivel organizativo e integra al sistema de recursos humanos en el entorno con el que interacciona. En definitiva, condiciona y es condicionado por las políticas empresariales al incluirse también en el análisis su influencia sobre otros aspectos internos de la organización o sus efectos sobre el ambiente en el que se desarrollan. Así pues, podemos afirmar que la asunción básica de este enfoque reside en que su objeto de investigación excede las decisiones empresariales así como la necesidad de incluir el entorno en los modelos explicativos y no tanto como una variable contingente.

Cuadro N..$^{\circ} 1$

Resumen de los enfoques o perspectivas de RRHH

\begin{tabular}{|c|c|c|}
\hline Enfoques & $\begin{array}{l}\text { Asunción } \\
\text { básica }\end{array}$ & $\begin{array}{c}\text { Relación } \\
\text { entre variables }\end{array}$ \\
\hline UNIVERSALISTA & Existencia de "best practices" & $\begin{array}{l}\text { Lineal, universal y } \\
\text { generalizable }\end{array}$ \\
\hline CONTINGENTE & $\begin{array}{l}\text { Se niega la existencia de "best } \\
\text { practices". La adopción de las } \\
\text { PRH dependerá de variables } \\
\text { como la estrategia corporativa } \\
\text { y el contexto interno y externo }\end{array}$ & $\begin{array}{l}\text { Las variables contingentes } \\
\text { (estrategia y entorno) } \\
\text { median la relación entre } \\
\text { la variable dependiente e } \\
\text { independiente. }\end{array}$ \\
\hline CONFIGURATIVO & $\begin{array}{l}\text { Es posible identificar patrones, } \\
\text { igualmente eficientes, en el } \\
\text { Sistema de RRHH que } \\
\text { provienen de la combinación } \\
\text { de elementos que construyen } \\
\text { el Sistema de RRHH }\end{array}$ & $\begin{array}{l}\text { Se introducen relaciones } \\
\text { interdependientes y } \\
\text { sinérgicas. Este enfoque se } \\
\text { centra en cómo los patrones } \\
\text { de RRHH afectan a la } \\
\text { performance empresarial }\end{array}$ \\
\hline CONTEXTUAL & $\begin{array}{l}\text { El SRH se considera de forma } \\
\text { más amplia. Es considerado } \\
\text { parte de un gran macro sistema } \\
\text { social, ambos influenciados y } \\
\text { capaces de influenciar la } \\
\text { estrategia de los RRHH }\end{array}$ & $\begin{array}{l}\text { Las variables se integran } \\
\text { en un supra-sistema }\end{array}$ \\
\hline
\end{tabular}

Fuente: Adaptado de Martín-Alcázar et al. (2005). 
Desde nuestro punto de vista, el enfoque contingente es el más adecuado a los entornos competitivos actuales ya que nos permite considerar tanto las variables estratégicas, como las organizativas y las relativas al entorno. Las variables estratégicas se refieren a que la aplicación de determinadas PRH depende de la idoneidad de la consistencia que exista entre las decisiones de RRHH y la estrategia corporativa (Martín-Alcázar et al., 2005). En segundo lugar, las variables organizativas se refieren al tamaño, tecnología, la estructura, o las relaciones de poder. Por último y referente a las variables del entorno pueden considerarse como tales los determinantes ambientales externos a la organización, como el entorno competitivo, tecnológico, macroeconómico y laboral.

De la adopción del enfoque contingente se desprende la necesidad o importancia de aplicar distintas PRH según la estrategia, por lo que se entiende que la adopción de estrategias orientadas a la innovación conlleva la aplicación de determinadas PRH. El análisis de esa temática se abordará con posterioridad en la sección 4.1 de este trabajo.

\section{Aspectos conceptuales, tipológicos y terminológicos de la innovación}

En este apartado se presentan los principales trabajos que analizan las distintas tipologías de innovación así como el análisis de las mismas y la selección de la tipología más adecuada para nuestro objeto de estudio.

En lo que a la innovación se refiere, cabe señalar que se trata de un término que abarca un fenómeno tan amplio como variado (Damanpour, 1992), susceptible de ser analizado y abordado desde múltiples ópticas, niveles y perspectivas. A pesar de la dificultad para definir este concepto, muchas son las definiciones que se le han dado sin llegar a ningún consenso por parte de la literatura por la dificultad de acotar este concepto. 
Las diferentes definiciones de innovación se agrupan en torno a dos grandes categorías: aquéllas que hablan de la innovación como un producto, y otras que la dotan de un sentido desde su carácter de proceso. Así, un ejemplo de la innovación de productos lo encontramos en el trabajo de Zaltman, Duncan y Holbeck (1973), los que la definen como alguna idea, práctica o material, percibido como nuevo por la relevante unidad de adopción. Por otra parte, Damanpour (1991) define la innovación en procesos como nuevos elementos, equipo o métodos, introducidos en el sistema productivo de la empresa o en las operaciones de servicios para elaborar un producto o prestar un servicio.

Este carácter producto-proceso se observa también en la definición proporcionada por Schumpeter a mediados del siglo pasado. Schumpeter (1942) propone cinco tipos de innovación: la introducción de un nuevo bien o el mejoramiento de un bien que ya existía, la introducción de un nuevo método de producción, la apertura de un nuevo mercado, la conquista de una nueva fuente de suministro de materias primas o bienes en proceso y la creación de un nuevo tipo de organización industrial.

A continuación se van a describir las principales tipologías sobre innovación, para seleccionar la más adecuada para nuestro marco teórico. De acuerdo con Anderson y King (1993) las áreas temáticas alrededor de las cuales se han identificado la mayor parte de las tipologías se refieren a: aproximación de los sistemas socio-técnicos, aproximación de las características de la innovación y aproximación a las fuentes de la innovación, tal y como queda recogido en el siguiente cuadro resumen: 
Prácticas de recursos humanos y su impacto en los resultados de innovación: Una revisión teórica

\section{Cuadro N. ${ }^{\circ} 2$ \\ Aproximación de los sistemas}

\begin{tabular}{|c|c|}
\hline Aproximaciones & Tipos de innovación \\
\hline $\begin{array}{l}\text { Aproximación de los } \\
\text { sistemas socio-técnicos } \\
\text { (Damanpour y Evan, } \\
\text { 1984) }\end{array}$ & $\begin{array}{l}\text { Administrativas: relacionadas con la estructura organizativa } \\
\text { y los procesos administrativos, es decir, innovaciones } \\
\text { relacionadas con el modo en que está organizado el } \\
\text { trabajo, las relaciones entre la gente que interactúa para } \\
\text { alcanzar una tarea u objetivo particular. } \\
\text { Técnicas estarían relacionadas con la actividad básica de } \\
\text { trabajo de una organización en términos de productos } \\
\text { y procesos, es decir, se corresponden con productos, } \\
\text { servicios y procesos. }\end{array}$ \\
\hline $\begin{array}{l}\text { Aproximación a las } \\
\text { características de la } \\
\text { innovación } \\
\text { (Anderson y King, } \\
\text { 1993). }\end{array}$ & $\begin{array}{l}\text { Programadas: fijadas o catalogadas de antemano. } \\
\text { No programadas: no fijadas o catalogadas de antemano. } \\
\text { Dentro de estas se diferencian tres tipos: } \\
\text { Slack Resources: derivadas de la escasa disponibilidad o } \\
\text { flexibilidad de recursos. }{ }^{1} \\
\text { Distress innovations: que tienen lugar cuando la organización } \\
\text { se enfrenta a una situación de crisis. } \\
\text { Innovaciones Proactivas: representadas por intentos de los } \\
\text { individuos para llamar la atención de las organizaciones } \\
\text { hacia áreas donde la posibilidad de cambio no había sido } \\
\text { contemplada. } \\
\text { Innovación Instrumental esencial: no necesitan de otras } \\
\text { que faciliten su introducción o desarrollo. } \\
\text { Radicalidad de la Innovación: relacionada con la novedad } \\
\text { y el riesgo; cuanto más nueva y arriesgada más radical. } \\
\text { Innovación incremental: surgen de los cambios progresivos } \\
\text { en productos y procesos para incrementar la eficiencia. }\end{array}$ \\
\hline $\begin{array}{l}\text { Aproximación a } \\
\text { las fuentes de la } \\
\text { innovación } \\
\text { (Anderson, 1989) }\end{array}$ & $\begin{array}{l}\text { Emergentes: generadas de forma espontánea sin finalidad } \\
\text { concreta. } \\
\text { Forzadas: generadas dentro de la organización represen- } \\
\text { tando respuestas únicas, inéditas o improbadas hacia los } \\
\text { desafíos y problemas particulares de una organización, } \\
\text { adoptadas o importadas desde fuera de la organización, e } \\
\text { innovaciones impuestas. }\end{array}$ \\
\hline
\end{tabular}

Fuente: Elaboración propia a partir de la revisión de Martín et al., 1997.

1 Nohria y Gulati (1996) definen slack resources como los recursos de una organización que exceden el mínimo necesario para producir un determinado output. Se incluyen trabajadores con tiempo libre, capacidad no utilizada, gastos e inversiones innecesarios. La empresa podría utilizarlos para responder a contingencias o para experimentar. 
La tipología plasmada en el Manual de Oslo (OECD-Eurostat, 2005) en su tercera edición, se distinguen cuatro tipos de innovación: las de producto, las de proceso, innovaciones de mercadotecnia y las de organización. Las innovaciones de producto y proceso están relacionadas estrechamente con el concepto de innovación tecnológica de producto e innovación tecnológica de proceso. Estas definiciones anteriores, junto a las explicaciones que se ofrecen en el Manual de 2005 parecen ajustarse en mayor medida a la realidad actual en comparación con las tipologías ofrecidas en el cuadro anterior, por lo que en este trabajo es conveniente adoptar parcialmente la tipología propuesta por el Manual de Oslo (2005) (OECD-Eurostat, 2005). A continuación se muestra un cuadro resumen con los conceptos y tipologías de innovación considerados relevantes para la realización de este trabajo.

\section{Cuadro N. ${ }^{\circ} 3$ \\ Conceptos y definiciones}

\begin{tabular}{ll}
\hline \multicolumn{1}{c}{ Concepto } & \multicolumn{1}{c}{ Descripción } \\
\hline Innovación & $\begin{array}{l}\text { La introducción de un nuevo, o significativamente } \\
\text { mejorado, producto (bien o servicio), de un proceso, } \\
\text { de un nuevo método de comercialización o de un } \\
\text { nuevo método organizativo, en las prácticas internas } \\
\text { de la empresa, la organización del lugar de trabajo o } \\
\text { las relaciones exteriores. }\end{array}$ \\
Innovación de & $\begin{array}{l}\text { Introducción de un bien o de un servicio nuevo, o } \\
\text { produificativamente mejorado, en cuanto a sus } \\
\text { características o en cuanto al uso al que se destina. }\end{array}$ \\
\hline $\begin{array}{l}\text { Innovación de } \\
\text { proceso }\end{array}$ & $\begin{array}{l}\text { Introducción de un nuevo, o significativamente } \\
\text { mejorado, proceso de producción o de distribución. }\end{array}$ \\
\hline
\end{tabular}

Fuente: Elaboración propia a partir de OCDE-Eurostat (2005).

Para concluir con el marco conceptual del presente trabajo, la definición que a nuestro juicio es más completa y que se va adoptar es la publicada en el Manual de Oslo (2005) y que define 
la innovación como "la introducción de un nuevo, o significativamente mejorado, producto (bien o servicio), de un proceso, de un nuevo método de comercialización o de un nuevo método organizativo, en las prácticas internas de la empresa, la organización del lugar de trabajo o las relaciones exteriores" (2005: 46).

De acuerdo con la definición ofrecida, una innovación debe tener alguno de los siguientes requisitos: generación y comercialización de nuevos productos o procesos, cambios en el Management y cambios sociales en relación a los RRHH en la organización.

\section{Prácticas de recursos humanos, estrategia y resultados de innovación}

\subsection{PRÁCTICAS DE RECURSOS HUMANOS Y ESTRATEGIA DE INNOVACIÓN}

Si comparamos los enfoques de innovación y de RRHH considerados en los puntos anteriores, a primera vista puede parecer que existen mayores diferencias que similitudes. Sin embargo, si profundizamos pueden detectarse conexiones que nos permiten crear un enfoque integrado (Leede y Kees, 2005).

La investigación en temas de innovación se ha centrado, generalmente, en los procesos, mientras que la investigación en RRHH se ha centrado en analizar el impacto de la estrategia corporativa sobre las PRH y estas sobre los resultados organizativos. Si consideramos las diferencias entre ambos enfoques podemos afirmar que el enfoque de RRHH es un poco más estático que el de innovación, ya que se centra más en analizar los resultados u outputs. Mientras el enfoque de innovación tiene un carácter integrador, dinámico y de cambios, el enfoque de RRHH se ha centrado en analizar relaciones causales entre PRH y resultados, otorgando poco valor a los procesos y al carácter dinámico y de cambio. 
La adopción de una estrategia corporativa conlleva la aplicación de determinadas PRH, o como afirman Schuler y Jackson (1987a) de determinados roles o comportamientos requeridos. Por lo que la adopción de una estrategia de innovación tiene importantes implicaciones para la Dirección de Recursos Humanos, ya que este tipo de estrategia requiere a personas trabajando de forma diferente (Schuler y Jackson (1987a). Estos autores afirman la necesidad de implementar seis PRH en las organizaciones que siguen una estrategia de innovación: trabajo en equipo, evaluaciones del rendimiento en función de objetivos grupales y con orientación a largo plazo, trabajos que fomenten el desarrollo personal y la promoción, sistemas de recompensa basados en la equidad interna más que en la externa, existencia de composición variable en los salarios y existencia de planes de desarrollo de carrera profesional.

Otros trabajos (Jackson et al., 1989; Jackson y Schuler, 1995; Schuler y Jackson, 1987a; 1987b; 1988; Tang, 1998) señalan que los modelos de innovación expresan la necesidad de aplicar políticas y prácticas concretas de RRHH para el desarrollo de habilidades, conocimientos y comportamientos orientados a la innovación. Por lo que consideramos de gran relevancia la adecuada integración entre las PRH y la estrategia corporativa de la organización y, en particular, la estrategia de innovación. En esta misma línea, Brockbank (1999) relaciona las PRH con la estrategia corporativa, y define y describe las PRH que contribuyen a crear y mantener una ventaja competitiva sostenible a través de la estrategia de innovación.

Analizadas las diferencias entre los enfoques y tras la consideración de la importancia del ajuste entre la estrategia corporativa y las PRH, cabe señalar que en el siguiente subapartado se va a tratar de relacionar las variables que se incluyen en las PRH y los resultados de innovación así como una revisión de los trabajos más representativos que han analizado esta relación. 


\subsection{PrÁcticas DE RECURSOS humanos y RESUltados DE INNOVACIÓN}

Como se señaló al inicio de este trabajo, cabe destacar la gran importancia concedida a la capacidad de innovar de las empresas, aunque con poca consideración desde la Gestión de los Recursos Humanos (Kang, Morris, y Snell, 2007). Tras la revisión de la literatura realizada y considerando los planteamientos de otros trabajos previos. Podemos afirmar que existen pocos estudios que tengan en cuenta el efecto de las $\mathrm{PRH}$ sobre los resultados de innovación (Chen y Huang, 2009).

Therrien y Leonard (2003) investigaron el impacto de las PRH como factores influyentes en la novedad de la innovación en empresas canadienses. Ellos encontraron que las PRH afectan la probabilidad de ser innovador. Por otra parte, estos autores afirman que las empresas que utilizan prácticas de participación de los trabajadores, formación o sistemas de recompensas son más propensas a ser first innovators. Además, la agrupación de estas prácticas en sistemas coherentes y jerárquicos también aumenta la probabilidad de ser un innovador relevante.

Por otra parte, Laursen y Foss (2003) desarrollaron el argumento de que así como la complementariedad entre las PRH influyen en el rendimiento financiero, también esta podría influir en los resultados de innovación. Estos autores examinaron esta cuestión mediante un estudio empírico utilizando datos daneses. Entre sus conclusiones señalaban que las PRH favorecen el desarrollo, la capacidad de innovación y el logro de resultados innovadores de la empresa, por ejemplo, informando a los empleados de que la actividad innovadora será reconocida y recompensada, facilitando la colaboración entre empleados a través del trabajo en equipo, procesos de participación y comunicación interna.

Pini y Santangelo (2005) estudiaron el impacto de la utilización de prácticas (verticalizadas) de RRHH, la existencia de diferentes 
formas de organizar la investigación y el desarrollo y la naturaleza de las competencias de los empleados, en la probabilidad de generar diferentes tipos de innovaciones. Los resultados de su estudio empírico para una muestra de empresas ubicadas en una provincia italiana, evidenció que el desarrollo de la innovación es una actividad heterogénea entre empresas nacionales y extranjeras (en Italia) como muestran los diferentes determinantes de la innovación (producto, proceso y calidad).

Autores como Shipton y otros (2005; 2006 a y b) estudiaron la relación entre las políticas o prácticas y la innovación. En estos estudios lo que se sugiere es que la innovación va a ser promovida y sostenida en la medida en que la empresa desarrolla una gestión de recursos humanos que refuerza las diferentes etapas del proceso de innovación. Concretamente, en el estudio longitudinal realizado en 2005 se comprueba empíricamente los efectos de la introducción de enfoques sofisticados para las prácticas de reclutamiento, selección, formación y evaluación para predecir resultados de innovación de productos y de tecnología de producción. Es así como se descubre la existencia de una relación positiva entre las $\mathrm{PRH}$, la innovación de producto y de tecnología de producción. Además, comprueban que la innovación se realza bajo un clima de aprendizaje y se inhibe cuando hay un vínculo entre la evaluación del rendimiento y la remuneración.

Los resultados obtenidos para el estudio de 2006 (Shipton et al., 2006a) revelan que la capacitación, la socialización, el trabajo en equipo, la evaluación y un enfoque exploratorio de aprendizaje, son los factores predictivos de la innovación para un conjunto de 22 empresas manufactureras del Reino Unido. Las recompensas en función del rendimiento, en combinación con un enfoque orientado al aprendizaje exploratorio, se asocian positivamente con la innovación en los sistemas técnicos.

En un estudio que también desarrolló Shipton junto con sus colaboradores (2006 b) se puso de manifiesto que la satisfacción 
laboral global fue un predictor significativo de la innovación organizativa posterior, incluso después de controlar la innovación anterior de la organización y la rentabilidad. Por otra parte los datos indicaron que la relación entre la satisfacción laboral global y la innovación en la tecnología de producción y procesos fue moderado por dos factores: la variedad de empleos y el compromiso de "single status/ harmonization".2

Con posterioridad, Sjoerd Beugelsdijk (2008) estudió la relación entre algunas PRH (formación y rotación de la tarea, autonomía, flexibilidad horaria, sistema retributivo en función del rendimiento, tipo de contrato de trabajo) y la innovación de productos. Algunos de los resultados más relevantes presentados en este estudio determinan que mientras la innovación incremental puede ser organizada a través de sistemas de incentivos y programas de formación, para promover las innovaciones radicales lo mejor es proporcionar autonomía a los trabajadores en cuanto a tareas y su planificación. De esta forma se establece una relación positiva entre algunas de las PRH y los resultados en la innovación de productos, utilizando la teoría de la creatividad como marco. Los resultados confirman la predicción teórica de que las prácticas de RRHH pueden ser un recurso valioso para las empresas que quieran innovar.

Por su parte, Jiménez y Sanz (2008) analizaron empíricamente la relación entre la innovación y la gestión de recursos humanos y el efecto sobre los resultados de la empresa. Sus hallazgos muestran que la innovación contribuye positivamente al rendimiento del negocio y que la gestión de recursos humanos (el diseño de trabajo flexible, el trabajo en equipo, la planificación de plantilla -desarrollo profesional-, la formación, la evaluación del rendimiento y los sistemas de recompensas) potencia la innovación.

2 Estos términos se refieren a situaciones donde hay equidad en las condiciones de trabajo y en aspectos no dinerarios. 
Otros estudios como el de Ortín y Santamaría (2009) se han ocupado -a través de un estudio de casos- del análisis de las PRH en los departamentos de $\mathrm{I}+\mathrm{D}+\mathrm{i}$ y la adaptación alcanzada en diferentes empresas. Los datos han mostrado que ciertas prácticas como el reclutamiento y la organización en el trabajo se han de adaptar al personal del departamento de $\mathrm{I}+\mathrm{D}+\mathrm{i}$. Los hallazgos de este estudio apoyan la idea de que debido a la madurez de los departamentos de $\mathrm{I}+\mathrm{D}+\mathrm{i}$, las actividades de investigación y desarrollo requieren trabajadores más cualificados y estimulados así como la creación de redes y equipos multidisciplinares. Así mismo, estos autores concluyen afirmando que la delegación de las PRH en el departamento de I+D+i mejora el grado de adaptación de las prácticas a las necesidades concretas del mismo.

En esta misma línea de investigación, Petra de Saá (2010) realizó un estudio cuyo objetivo consistía en identificar los factores internos de RRHH (la existencia de un plan de RRHH y la estabilidad en el trabajo) que podían afectar a la capacidad de innovación de las empresas periféricas de la Unión Europea. A través de la realización de un estudio empírico (127 empresas canarias) se llegó a la conclusión de que la Dirección de Recursos Humanos de alto compromiso (reclutamiento y selección, promoción, evaluación para la promoción, participación, plan de formación, retribución variable, seguridad en el trabajo) tiene una influencia positiva sobre la innovación en los procesos de la organización. Los resultados también muestran que la formalización de las PRH en un plan y la estabilidad en el empleo también incrementan la innovación en los procesos.

Si consideramos los estudios publicados más recientemente, Cheng y Mohd (2010) centraron su análisis en examinar la relación entre la gestión de RRHH (evaluación del desempeño, desarrollo profesional, capacitación, sistema de recompensas y el reclutamiento) y capacidad de innovación (de producto, de procesos y administrativa) de la organización, destacando el profundo papel 
Prácticas de recursos humanos y su impacto en los resultados de innovación: Una revisión teórica

que desempeña la estimulación de la formación en la innovación organizativa en el contexto de las empresas manufactureras de Malasia para los tres tipos de innovación considerados.

A modo de síntesis, la tabla N. ${ }^{\circ} 4$ recoge de manera concreta la importancia que cada una de las PRH ha tenido en el estudio de la relación entre PRH y resultados de innovación.

\section{Cuadro N. ${ }^{\circ} 4$}

Importancias de las PRH en los trabajos que han estudiado la relación entre las PRH y los resultados de innovación

\section{Prácticas \\ Estudios empíricos relevantes}

\begin{tabular}{|c|c|}
\hline Autonomía & $\begin{array}{l}\text { Beugelsdijk, 2010; Laursen y Foss, 2003; Ortín y } \\
\text { Santamaría, } 2009\end{array}$ \\
\hline Participación & $\begin{array}{l}\text { Therrien y Leonard, 2003; Laursen y Foss, 2003; Jiménez } \\
\text { y Sanz, 2008; Cheng y Huang, 2009; Saa y Díaz, } 2007\end{array}$ \\
\hline Capacitación & $\begin{array}{l}\text { Lau y Ngo, 2004; Beugelsdijk, 2010; Therrien y Leonard, } \\
\text { 2003; Laursen y Foss, 2003; Shipton et al, 2005, 2006a; } \\
\text { Jiménez y Sanz, 2008; Cheng y Huang, 2009; Saa y } \\
\text { Díaz, 2007; Cheng y Mohd, 2010; Searle y Ball, } 2003\end{array}$ \\
\hline Sistemas de recompensas & $\begin{array}{l}\text { Lau y Ngo, 2004; Searle y Ball, 2003; Therrien y Leonard, } \\
\text { 2003; Ortín y Santamaría, 2009; Cheng y Huang, 2009; } \\
\text { Saa y Díaz, 2007; Cheng y Mohd, } 2010\end{array}$ \\
\hline $\begin{array}{l}\text { Remuneración vinculada a } \\
\text { los resultados }\end{array}$ & $\begin{array}{l}\text { Beugelsdijk, 2010; Laursen y Foss, 2003; Shipton et al, } \\
\text { 2005; Shipton et al. 2006 a; Jiménez y Sanz, 2008; } \\
\text { Cheng y Huang, 2009; Cheng y Mohd, } 2010\end{array}$ \\
\hline Trabajo en equipo & $\begin{array}{l}\text { Lau y Ngo, 2004; Pini y Santangelo, 2005; Shipton et al, } \\
2006 \text { a; Jiménez y Sanz, 2008; Ortín y Santamaría, } 2009\end{array}$ \\
\hline Rotación de tareas & $\begin{array}{l}\text { Beugelsdijk, 2010; Laursen y Foss, 2003; Pini y Santangelo, } \\
\text { 2005; Shipton et al, } 2006 \text { b; Ortín y Santamaría, } 2009\end{array}$ \\
\hline Reclutamiento y selección & $\begin{array}{l}\text { Shipton et al, 2005; Ortín y Santamaría, 2009; Saa y } \\
\text { Díaz, 2007; Cheng y Mohd, 2010; Searle y Ball, 2003; } \\
\text { Subramaniam y Youndt, } 2005\end{array}$ \\
\hline $\begin{array}{l}\text { Oportunidades de desarrollo } \\
\text { de carrera profesional }\end{array}$ & $\begin{array}{l}\text { Jiménez y Sanz, 2008; Ortín y Santamaría, 2009; Saa y } \\
\text { Díaz, 2007; Cheng y Huang, 2009; Cheng y Mohd, } \\
2010\end{array}$ \\
\hline
\end{tabular}

Fuente: Elaboración propia. 
Podemos afirmar que el conocimiento sobre la contribución de la gestión de los recursos humanos, a través de las PRH, a los resultados de innovación es aún muy escaso, por lo que se requiere una mayor atención a esta relación (Laursen y Foss, 2003; Shipton et al., 2006a).

\subsection{OTRAS VARIABLES A CONSIDERAR}

Tras la revisión de la literatura se ha observado que diversos son los trabajos que consideran otras variables que pueden afectar la relación entre las PRH y los resultados de innovación. En este apartado, lo que se pretende es destacar aquellas más importantes y que por lo tanto hay que considerar en futuros análisis.

Cheng y Huang (2009) examinan el papel de la capacidad de la gestión del conocimiento en la relación entre la planificación estratégica de las PRH y la innovación desde el punto de vista del conocimiento (Knowledge based view). Los resultados indicaron que las PRH se relacionan positivamente con la capacidad de gestión del conocimiento y que, al mismo tiempo, tienen un efecto positivo en el rendimiento de la innovación. Los resultados proporcionaron evidencia de que la capacidad de gestión del conocimiento desempeña un papel mediador entre la planificación estratégica de las PRH y la innovación.

Por su parte, Wei, Li-Qun, Liu, Jun y Herndon (2011) estudian la relación entre el sistema de RRHH y la innovación de productos. Siguiendo la perspectiva contextual, los autores examinan un modelo interactivo en el que se propone la cultura corporativa y la estructura organizativa como variables moderadoras de la relación entre las PRH y la innovación de productos. Los resultados empíricos de 223 empresas chinas indican que el sistema de RRHH tiene un impacto positivo en la innovación de productos y, además, esta relación es más fuerte para las empresas con una cultura orientada al desarrollo. De igual modo, el examen de las interacciones indica que en las empresas con una estructura más 
horizontal, la relación entre las PRH y la innovación es más fuerte con una cultura orientada al desarrollo (Wei et al., 2011).

Por último, Jiang, Wang, y Zhao (2012) utilizando una muestra de 106 empresas chinas examinaron cómo las PRH estaban relacionadas con la creatividad de los empleados y con la innovación organizativa. Los resultados mostraron que prácticas como el reclutamiento y la selección, el sistema de recompensas, el diseño del trabajo y el trabajo en equipo, están vinculadas positivamente con la creatividad. Sin embargo, prácticas como la capacitación y la evaluación del rendimiento no. En este trabajo la creatividad mediaba la relación entre estas cuatro PRH y la innovación organizativa. Estos resultados sugieren que las PRH juegan un papel importante a la hora de promover la innovación en las empresas chinas.

A modo de síntesis, existen otras variables organizativas que pueden tener impacto entre las PRH y los resultados de innovación y que por lo tanto deben ser incluidas en los análisis para obtener un mejor entendimiento de la realidad. En este sentido, variables como la capacidad de gestión del conocimiento y la creatividad del empleado podrían actuar como mediadoras entre las PRH y los resultados de innovación, mientras la cultura corporativa y la estructura organizativa podrían asumir un rol moderador.

\section{Conclusiones}

La contribución básica de esta investigación reside en la revisión exhaustiva de la literatura que estudia la relación entre las PRH y los resultados de innovación. A modo de conclusión, se ha planteado un modelo teórico que se explica en este apartado (véase la figura $\mathrm{N} .{ }^{\circ} 1$ ).

Desde nuestro punto de vista, la integración de estos dos campos (PRH y resultados de innovación) solo es posible si desde la 
organización se adopta una estrategia de innovación (no solo de costes o diferenciación) y termina con resultados organizativos tales como la producción de nuevos productos y servicios, la implementación de nuevos procesos... etc.

Figura N. ${ }^{\circ} 1$

\section{Modelo teórico}

\section{PRH}

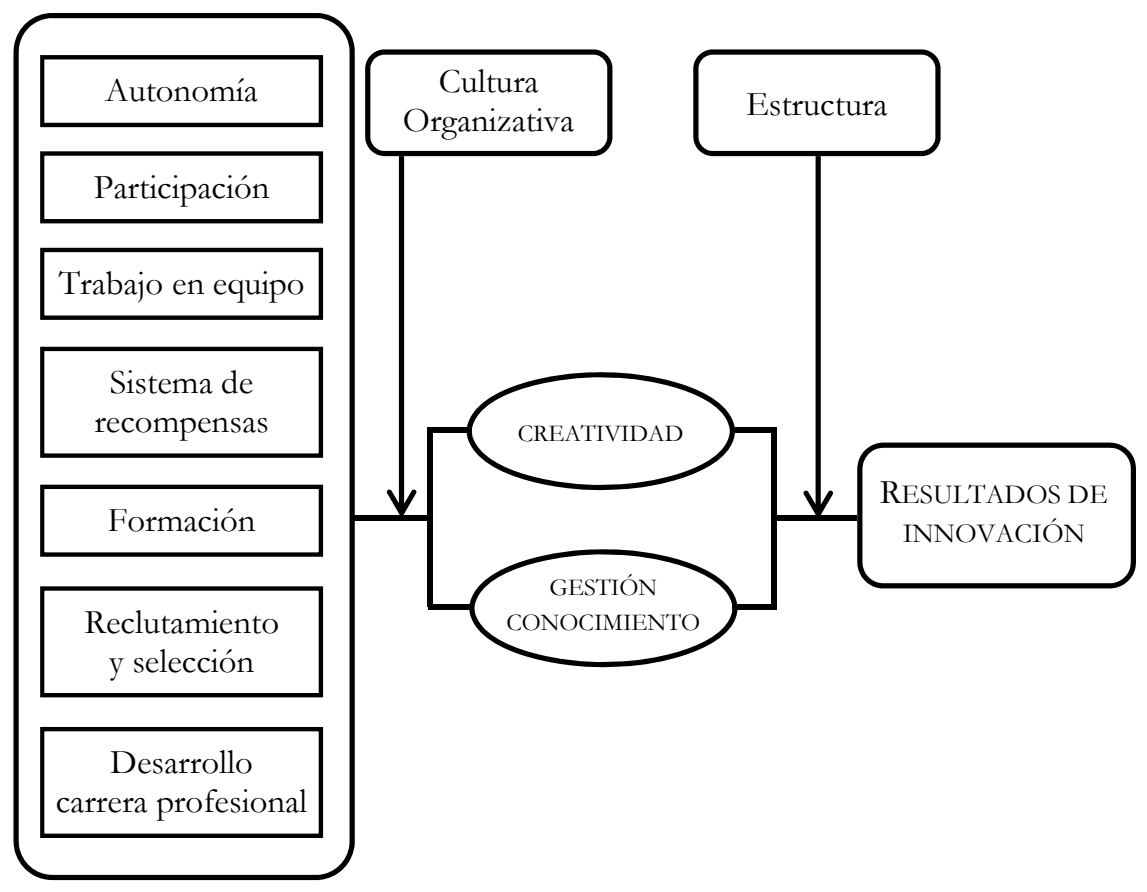

Para dar respuesta a nuestro primer objetivo (tras el análisis de la literatura, la identificación de las PRH más analizadas en cuanto a los resultados de innovación) podemos concluir afirmando que desde la literatura las PRH más analizadas se refieren a los siguientes términos: la autonomía, participación, formación, sistemas de recompensas, trabajo en equipo, reclutamiento y la selección, además del desarrollo de carrera profesional. El modelo 
teórico, presentado en la figura $\mathrm{N} .^{\circ} 1$, ilustra la relación entre los distintos constructos de acuerdo con la revisión de la literatura. Respecto a las PRH, la autonomía, la participación, la formación, los sistemas de recompensas, el trabajo en equipo, el reclutamiento y la selección, y el desarrollo de carrera profesional, afectarían positivamente los resultados de innovación a través de la creatividad de los empleados y la gestión del conocimiento. Además, según la literatura especializada la cultura organizativa y la estructura de la organización moderan la relación entre las PRH y los resultados de innovación, por lo que en relación a nuestros objetivos estas variables también tienen impacto en la relación analizada y por lo tanto, se han incluido.

Tras las principales conclusiones extraídas, el estudio realizado lleva aparejado implicaciones tanto académicas como prácticas. Respecto a las implicaciones prácticas cabe señalar que es condición indispensable la adopción de unas PRH determinadas cuando se lleva a cabo una estrategia orientada a la innovación. Se debe espolear la creatividad de los empleados para innovar fomentando la autonomía, la participación, la formación, los sistemas de recompensas, el trabajo en equipo, el reclutamiento y la selección y el desarrollo de carrera profesional. Estos procesos han de estar orientados a retener empleados y dotarlos de capacidades heterogéneas y flexibles a través del continuo proceso de aprendizaje. Además, variables como la estructura y la cultura organizativa tienen efectos sobre la relación entre las PRH y los resultados de innovación. Como han señalado autores como Wei y otros (2011) con una estructura más horizontal y con una cultura orientada al desarrollo, la relación entre las PRH y los resultados de innovación es más fuerte.

Respecto a las implicaciones académicas, en primer lugar, se aporta a la literatura de recursos humanos un enfoque más dinámico en el estudio de las PRH vinculadas a los resultados de innovación y al enfoque de innovación orientándolo hacia los resultados. 
Además, con el planteamiento del modelo teórico se introducen nuevas variables que moderan y median la relación entre las PRH y los resultados de innovación como la creatividad, que según la literatura especializada han sido estudiadas de forma insuficiente (Jiang et al., 2012). Por lo que la inclusión de estas variables contextuales (cultura y estructura organizativa) se abren a nuevas perspectivas para analizar la relación entre las PRH y los resultados de innovación.

Respecto a las limitaciones que conciernen al presente trabajo básicamente residen en que se trata de un trabajo meramente teórico, en el que a pesar de haber revisado los principales trabajos del área en que se enmarca el objeto de estudio, la revisión de la literatura es limitada dadas las restricciones de espacio. Otra limitación se refiere a que planteamos un modelo amplio y unas proposiciones implícitas que cobrarán valor cuando sean contrastadas empíricamente, a través de un análisis cuantitativo o cualitativo y se muestre evidencia empírica respecto de estas relaciones.

Este estudio es una aproximación teórica al objeto de análisis. En un futuro debe desarrollarse un modelo que permita analizar relaciones causales entre los diferentes constructos, incluyendo también otros resultados empresariales (rentabilidad, crecimiento de las ventas, cuota de mercado) y los resultados frente a la sociedad, en términos de posicionamiento e imagen.

De acuerdo con Klijn y Tomic (2010) es necesaria una mayor atención a la comparación de información entre los diferentes campos de estudio que analizan la creatividad para desarrollar mejores teorías que especifiquen los determinantes y promotores de la creatividad. También se requiere una mayor actividad investigadora en cómo transformar ideas creativas de forma eficiente en resultados innovadores y que estos se mantengan en organizaciones que operen en entornos turbulentos e inestables. 
Por otra parte, otra futura línea de investigación tendría en cuenta la relación entre las PRH e innovación a diferentes niveles (individual, grupal y organizativo) para enriquecer los resultados, es decir, estudiar el efecto de las PRH entre diferentes grupos de trabajo y los resultados de la innovación para observar las diferencias intra-grupo e inter-grupo de la percepción de las PRH y sus efectos sobre los resultados.

Por último, de la realización de este estudio y de la revisión del trabajo de Ortín y Santamaría (2009) nos hemos planteado la necesidad o no de adaptar las PRH a los departamentos de $\mathrm{I}+\mathrm{D}+\mathrm{i}$ para obtener mayores resultados de innovación. Estos autores afirman que las PRH se han de adaptar y desde el segundo caso del presente estudio se insiste en que no se han de adaptar por lo que proponemos el estudio para determinar si la adaptación o no de las PRH conlleva la obtención de mayores resultados de innovación.

\section{Referencias bibliográficas}

Anderson, N. (1989). Work group innovation: Current research concerns and future directions. (Comunicación presentada al IV Congreso de Psicología del Trabajo y de la Organización). Universidad de Cambrigde, Inglaterra.

Anderson, N. y King, N. (1993). Innovation in organizations. International Review of Industrial and Organizational Psychology, 8,1-34.

Becker, B., y Gerhart, B. (1996). The impact of human resource management on organizational performance: Progress and prospects. Academy of Management Journal, 39(4), 779-801.

Beugelsdijk, S. (2008). Strategic human resource practices and product innovation. Organization Studies, 29(6), 821-847. 
Brewster, C. (1995). Towards a european model of human resource management. Journal of International Business Studies, 26(1), 1-21.

Brewster, C. (1999). Strategic human resource management: The value of different paradigms. Management International Review, 39, 45-64.

Brockbank, W. (1999). If HR were really strategically proactive: Present and future directions in HR's contribution to competitive advantage. Human Resource Management, 38(4), 337-352.

Camelo, C., Martín, F., Romero, P. M., y Valle, R. (2004). Human resources management in spain: Is it possible to speak of a typical model? The International Journal of Human Resource Management, 15(6), 935-958.

Chen, C., y Huang, J. (2009). Strategic human resource practices and innovation performance - The mediating role of knowledge management capacity. Journal of Business Research, 62(1), 104-114.

Cheng, T., y Mohd, A. (2010). Human resource management practices and organizational innovation: An empirical study in Malaysia. The Journal of Applied Business Research, 26(4), 105-115.

Damanpour, F. (1991). Organizational innovation: A meta-analysis of effects of determinants and moderators. Academy of Management Journal, 34(3), 215-240.

Damanpour, F. (1992). Organizational Size and Innovation. Organization Studies, 13(3), 257-279. 
De Cieri, H., \& Krammar, R. (2008). In North Ryde N. S. W. (Ed.), Human resource management: Strategy, people, performance. Australia Pty Ltd: McGraw-Hill.

De Saá-Pérez, P., \& Díaz-Díaz, N. L. (2010). Human resource management and innovation in the Canary islands: An ultraperipheral region of the european union. The International Journal of Human Resource Management, 21(10), 1649-1666.

Delery, J. E., y Doty, D. H. (1996). Modes of theorizing in strategic human resource management: Tests of universalistic, contingency, and configurational performance predictions. Academy of Management Journal, 39(4), 802.

Dessler, G. (2011). Human resource management (12va ed.) PrenticeHall.

Gurbuz, S., \& Ibrahim, S. M. (2011). Impact of the strategic human resource management in organizational performance: Evidence from turkey. The International Journal of Human Resource Management, 22(8), 1803-1822.

Huselid, M. (1995). The impact of human resource management practices on turnover, productivity and corporate financial performance. Academy of Management Journal, 38, 635-672.

Jackson, S. E., \& Schuler, R. S. (1995). Understanding human resource management in the context of organizations and their environments. Annual Review of Psychology, 46, 237-264.

Jackson, S. E., Schuler, R. S., \& Rivero, J. M. (1989). Organizational characteristics as predictors of personnel practices. Personnel Psycology, 42, 727-786. 
Jiang, J., Wang, S., \& Zhao, S. (2012). Does HRM facilitate employee creativity and organizational innovation? A study of chinese firms. International Journal of Human Resource Management, 23(19), 4025-4047.

Jiménez-Jiménez, D., \& Sanz-Valle, R. (2008). Could HRM support organizational innovation? The International Journal of Human Resource Management, 19(7), 1208-1221.

Kang, S., Morris, S. S., \& Snell, S. A. (2007). Relational archetypes, organizational learning, and value creation: Extending the human resource architecture. The Academy of Management Review, 32(1), 236-256.

Klijn, M., \& Tomic, W. (2010). A review of creativity within organizations from a psychological perspective. The Journal of Management Development, 29(4), 322-343.

Laursen, K., \& Foss, N. J. (2003). New human resource management practices, complementarities and the impact on innovation performance. Cambridge Journal of Economics, $27,243-263$.

Leede, J. D., \& Kees, J. L. (2005). Innovation and HRM: Towards an integrated framework. Creativity and Innovation Management, 14(2), 108-117.

Looise, J., \& van Riemsdijk, M. (2004). Innovating Organizations and HRM: A Conceptual Framework. Management Revue, 15(3), 277-288.

Martín-Alcázar, F., Romero-Fernández, P. M., \& Sánchez-Gardey, G. (2005). Strategic human resource management: Integrating the universalistic, contingent, configurational and contextual perspectives. International Journal of Human Resource Management, 16(5), 633-659. 
Martín-Alcázar, F., Romero-Fernández, P. M., \& Sánchez-Gardey, G. (2004). Integración de las perspectivas universalista, contingente, configuracional y contextual en la investigación en dirección estratégica de los recursos humanos. Investigaciones Europeas de Dirección y Economía de la Empresa, 10(2), 29-54.

Miles, R. E., \& Snow, C. C. (1978). Organizational strategy, structure, and process. New York: McGraw-Hill.

Organization for economic co-operation and development statistical office of the European communities. (2015). Oslo manual: Guidelines for collecting and interpreting technological innovation data (3rd Ed.). Paris: OECD Publishing.

Ortín, P., \& Santamaría, L. (2009). R\&D managers' adaptation of firms’ HRM practices. R\&D Management, 39(3), 271-290.

Osterman, P. (1994). How common is workplace transformation and who adopts it?, 47, 173-188. Industrial and Labour Relations Review, 47, 173-188.

Pfeffer, J. (1994). Competitive advantage through people: Unleashing the power of the work force. Boston: Harvard Business School Press.

Pfeffer, J. (1998). Seven practices of successful organizations. California Management Review, 40(2), 96-124.

Pini, P., \& Santangelo, G. D. (2005). Innovation types and labour organizational practices: A comparison of foreign and domestic firms in the reggio Emilia Industrial Districts. Economics of Innovation and New Technology, 14(4), 251-276.

Schuler, R. S., \& Jackson, S. E. (1987a). Linking competitive strategies with human resource management practices. Academy of Management Executive, 1(3), 207-219. 
Schuler, R. S., \& Jackson, S. E. (1987b). Organizational strategy and organizational level as determinants of human resources practices. Human Resource Planning, 10(3), 125-141.

Schuler, R. S., \& Jackson, S. E. (1988). Linking remuneration practices to innovation as a competitive strategy. Human Resource Management Australia, 10(5), 6-21.

Schumpeter, J. A. (1942). Capitalism, Socialism and Democracy. New York: Harper \& Row.

Shipton, H., Fay, D., West, M. A., Patterson, M. G., \& Birdi, K. (2005). Managing people to promote innovation. Creativity and Innovation Management, 14(2), 118-128.

Shipton, H., West, M. A., Dawson, J. F., Birdi, K., \& Patterson, M. G. (2006a). HRM as a predictor of innovation. Human Resource Management Journal, 16(1), 3-27.

Shipton, H., West, M. A., Parkes, C. L., Dawson, J. F., \& Patterson, M. G. (2006b). When promoting positive feelings pays: Aggregate job satisfaction, work design features, and innovation in manufacturing organizations. European Journal of Work and Organizational Psychology, 15(4), 404-430.

Tang, H. K. (1998). An integrative model of innovation in organizations. Technovation, 18(5), 297-309.

Therrien, P., \& Léonard, A. (Ed.). (2003). In Otawa: Statistics. Empowering employees: A route to innovation. Canada: Statistics Canada; Human Resources Development Canada.

Venkatraman, N., \& Prescott, J. E. (1990). Environmentstrategy coalignment: An empirical test of its performance implications. Strategic Management Journal, 11(1), 1-23. 
Vlachos, I. (2008). The effect of human resource practices on organizational performance: Evidence from greece. The International Journal of Human Resource Management, 19(1), 74-79.

Wei, L., Liu, J., \& Herndon, N., C. (2011). SHRM and product innovation: Testing the moderating effects of organizational culture and structure in chinese firms. The International Journal of Human Resource Management, 22(1), 19-33.

Wimbush, J. C. (2005). Spotlight on human resource management. Business Horizons, 48(6), 463-467.

Zaltman, G., Duncan, R. \& Holbek, J. (1973) Innovations and Organizations. New York: Wiley. 


\section{Naiara Escribá Carda}

Tiene una maestría en Estrategia de Empresas; licenciada en Ciencias del Trabajo y diplomada en Relaciones Laborales por la Universidad de Valencia, España. En esta misma institución realiza su doctorado en Dirección de Empresas; forma parte del personal investigador en formación y se desempeña como docente en el departamento de Dirección de Empresas "Juan José Renau Piqueras" adscrito a la Facultad de Economía de la Universidad de Valencia. Sus intereses de investigación versan sobre la relación entre las prácticas de recursos humanos y los resultados de innovación y el papel que juega la creatividad de los empleados en dicha relación.

Email: Naiara.Escriba@uv.es

\section{Francisco Balbastre Benavent}

Es licenciado en Ciencias Económicas y Empresariales y doctor en Ciencias Económicas y Empresariales por la Universidad de Valencia, España. Ha sido director del máster en Dirección Estratégica de Organizaciones Deportivas de la Universidad de Valencia; vice-secretario de Calidad de la Facultad de Economía y co-director del Máster Oficial en Gestión de Calidad de dicha Universidad. Es profesor titular de Organización de Empresas en la Universidad de Valencia y su especialización docente e investigadora se centra en áreas relacionadas con el estudio de la 
implantación de sistemas de calidad y modelos de excelencia empresarial, el aprendizaje organizativo y la gestión del conocimiento, la formación de la estrategia y el proceso de innovación en Pymes.

Email: francisco.balbastre@uv.es

\section{Teresa Canet Giner}

Es licenciada en Ciencias Económicas y Empresariales; doctora en Ciencias Económicas y Empresariales por la Universidad de Valencia, España. En la actualidad es directora del Máster Oficial de Dirección y Gestión de Recursos Humanos, ofertado por la Universidad de Valencia. Su especialización docente e investigadora se enfoca en el área de la Dirección Estratégica y el Diseño Organizativo así como en la aproximación de ambas áreas al estudio de los diversos Modelos de Excelencia. Ha centrado también una parte de su investigación en los estudios de género en relación con la estrategia y la negociación, así como en el estudio de los procesos de formación de la estrategia en las Pymes.

Email: teresa.canet@uv.es 
